\title{
Developing an Approach for Fault Detection and Diagnosis of Angular Velocity Sensors
}

\author{
Hong Son Tran ${ }^{1}$, Dinh Dung Nguyen ${ }^{2}$, Thi Thuy Tran ${ }^{3}$, Dat Dang Quoc ${ }^{4}$, Hong Tien Nguyen ${ }^{5}$ \\ ${ }^{1}$ Le Quy Don Technical University, Hanoi, Vietnam \\ tranhongson@lqdtu.edu.vn - (iD) 0000-0002-7956-2377 \\ ${ }^{2}$ Budapest University of Technology and Economics, Department of Aeronautics, Budapest, Hungary \\ ddnguyen@vrht.bme.hu - (D) 0000-0002-8966-051X \\ ${ }^{3}$ Academy of Military Science and Technology, Hanoi, Vietnam \\ trthuy.mta@gmail.com - (D) 0000-0002-3586-8753 \\ 4 The Faculty of Control Engineering, Le Quy Don Technical University, Hanoi, Vietnam \\ dangquocdat201@gmail.com - (D) 0000-0002-7878-6379 \\ ${ }^{5}$ Air Defence-Air force Academy, Hanoi, Vietnam \\ smallcat24829@gmail.com - (D) 0000-0003-2037-4520
}

\begin{abstract}
Angular velocity sensor detection and diagnosis become increasingly essential for the improvement of reliability, safety, and efficiency of the control system on aircraft. The classical methods for fault detection and diagnosis are limit or trend checking of some measurable output variables. Due to they do not give a deeper insight and usually do not allow a fault diagnosis, model-based methods of fault detection and diagnosis were developed by using input and output signals and applying dynamic process models. These approaches are based on parameter estimation, parity equations, or state observers. This paper presents an improvement method to build algorithm fault diagnosis for angular velocity sensors on aircraft. Based on proposed method, results of paper can be used in designed intelligent systems that can automatically fault detection on aircraft.
\end{abstract}

\section{Keywords}

\author{
Block sensor \\ Fault diagnosis \\ Fault detection \\ Angular velocity sensor \\ Advanced control system
}

Time Scale of Article

Received 5 March 2021

Revised to 4 June 2021

Accepted 8 June 2021

Online date 27 June 2021

\section{Introduction}

Recently, fault detection and diagnosis (FDD) have an essential role in obtaining fault tolerance of aircraft control systems (Xue et al., 2007; He et al., 2020). Many approaches have been proposed for sensor or actuator FDD (Chen et al. 2012; Isermann 2005; Lu et al. 2016). In aviation engineering, the FDD of sensors and actuators for fixed-wing aircraft widely studies can be found in references (Lu et al., 2015; Kim et al., 2008; Xue et al., 2007). Investigation of the FDD for unmanned aerial vehicles (UAV) can also be found in Baskaya et al. (2017), and Hajiyev et al. (2013).
In general, sensors provide information for the control system according to their characteristics to ensure that the control system works well. They need the angular velocity parameter in the three axes of the coordinate system. Angular rate sensors play an essential role in the control system. The sensors' accuracy affects the quality of control because it is a component in a loop that controls the angular position. These sensors' signals work as the reverse contact signals, which are essential signal components in building high-quality control systems. Its position in the flying equipment control system is shown in Fig.1. 


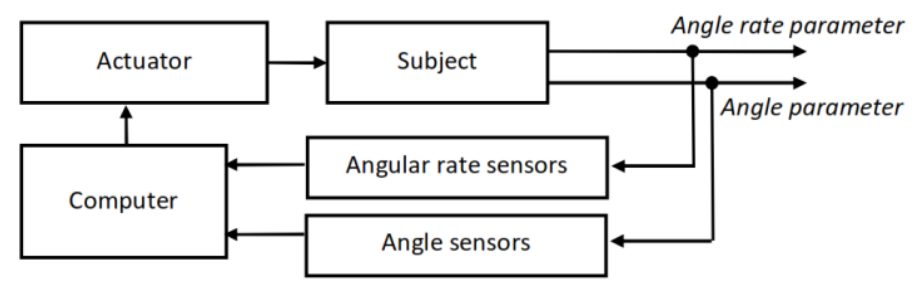

Fig. 1. Block diagram of flying equipment control

In Fig. 1, the aircraft is subject; the input parameter is angle deflection; the output parameters are the angular rate, angular position, including yaw, pitch, and roll angle. According to specific control laws, the computer functions to synthesize control signals to ensure the best quality for the control process of a specific flight mode.

A conventional angular speed sensor uses a gyroscope as the critical element to measure angular speed. They come in many different forms in terms of construction and accuracy, such as using a sensitive element that is a 2 -frame gyro or 3-frame gyro. The basic principle of an angular speed sensor is shown in the following figure.

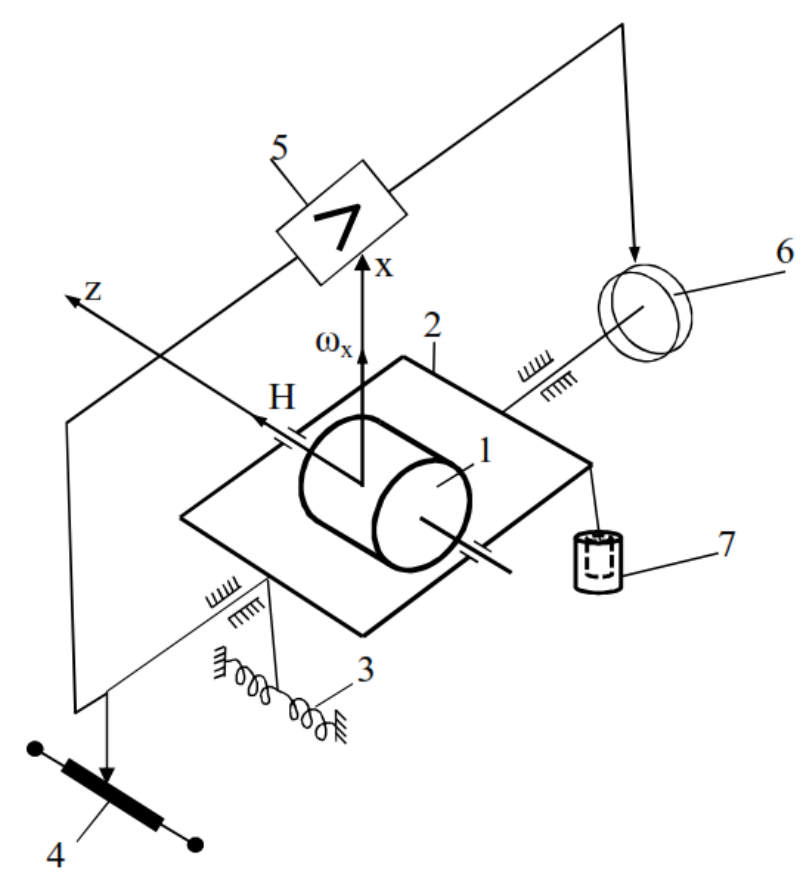

Fig. 2. Diagram of the principle of speed gyroscope

Where:

1. Gyro rotor.

2. Hanging frame.

3. Reactive springs.

4. Variable resistor.

5. Amplification.

6. Torque motor.

7. The damping mechanism.
When the gyro stand rotates around the Ox axis, the gyroscope performs two movements: a rotation around the rotor's axis and a rotation with the stand placing them around the measuring axis. Then there appears a gyro moment proportional to the angular speed vector of the gyro mount and the kinematic torque vector, causing the sling to tilt at an angle. Then the spring torque will produce a precession rate proportional to the angular speed to be measured. Typically, springs with linear characteristics are used, which means that the spring torque is proportional to the swing angle of the suspension frame relative to the angular speed measured by the inductance. In short, based on the precession properties of the gyroscope, the angular speed sensor measures the angular speed of the object. The layout of a single angular speed sensor is shown in the following figure.

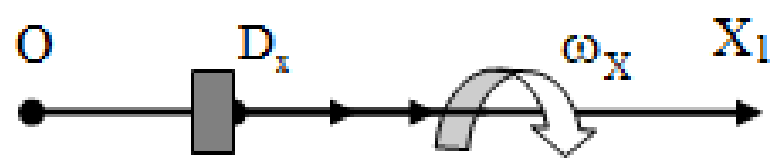

Fig. 3. Diagram of the principle of angular speed sensor on an axis

However, to ensure the safety of the flying object, angular speed sensors will be used with 1 to 4 sensors on each axis. On aircraft, the 3-axis scale of the standard coordinate system is $3-3-4$.

The actuator is responsible for receiving control signals, handling and deflecting the steering blades so that under the action of aerodynamic forces and torque, the flying equipment's state changes. Angular velocity and angle sensors are responsible for sensing control parameters and providing information for the computer to synthesize control laws. Thus, the angular rate sensors are in the control system's loop, which plays an essential role in improving control quality. The angular rate sensors arranged on the flying equipment correspond to the axes of the coordinate system $\mathrm{OX}_{1} \mathrm{Y}_{1} \mathrm{Z}_{1}$ in a 3-3-4 scale to ensure the redundancy of the sensors' information. However, with such a structure arrangement, the sensors can only provide angular rate information to the control system but cannot provide information about the operating quality of the sensors. According to the research results proposed in (Tuan et al. 2013), the author changed the layout of the angular speed sensors in the control system. There are nine angular rate sensors arranged in three axes of the coordinate system $\mathrm{OX}_{1} \mathrm{Y}_{1} \mathrm{Z}_{1}$ (3 sensors per axis) used to measure the roll rate $\omega_{x}$, yaw rate $\omega_{y}$, pitch rate $\omega_{z}$, respectively. The remaining sensor is used to determine the relationships between sensors. This sensor is arranged so that the sensitive axis coincides with the diagonal of the cube passing through the three axes, with 1 vertex being the origin $O$ of the coordinate system $\mathrm{OX}_{1} \mathrm{Y}_{1} \mathrm{Z}_{1}$ and a vertex on the OO' axis (the sensitive axis of sensor $\mathrm{S}_{0}$ ) as shown in Fig. 2. 


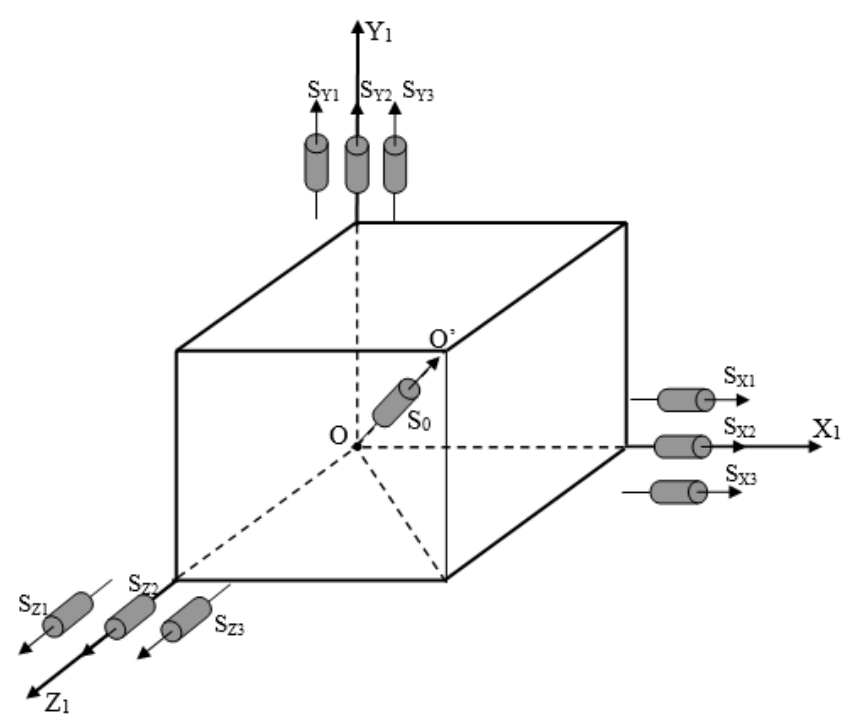

Fig. 4. Position of improved angular speed sensors on the three axes of the coordinate system

In general, we need to consider block sensors' error for diagnosing the fault of block sensors with its structure in fig 4.

The mathematical model of a sensor is given in the form:

$$
U_{D}(t)=k_{D} \omega(t)
$$

Where: $U_{D}(t)$ - Output voltage of the sensor;

$k_{D}$ - The amplification coefficient of the sensor;

If the sensors are working well, the output voltage is proportional to the angular speed value along the respective axes. However, if this sensor has errors, this mathematical model is not correct. The primary cause of failures is generally due to various defects and failures of the system's elements. Based on the actual operation of the sensors, these failures can be non-positive (negative) drift, increase (decrease) amplification factor, shortcircuit of sensors, breakage of sensors line. For each of these failures can be modelled in the form of a mathematical model as follows:

$$
\begin{aligned}
& \widetilde{U}_{D_{X}}(t)=k_{D_{X}} \omega_{X}(t)+\widetilde{U}_{D_{X_{0}}}(t) ; \widetilde{U}_{D_{X_{0}}}(t)<0 \\
& \widetilde{U}_{D_{X}}(t)=k_{D_{X}} \omega_{X}(t)+\widetilde{U}_{D_{X_{0}}}(t) ; \widetilde{U}_{D_{X_{0}}}(t)>0 \\
& \widetilde{U}_{D_{X}}(t)=\tilde{k}_{D_{X}} \omega_{X}(t) ; \tilde{k}_{D_{X}}=k_{D_{X}}+\Delta k_{D_{X}} ; \Delta k_{D_{X}}>0 \\
& \widetilde{U}_{D_{X}}(t)=\tilde{k}_{D_{X}} \omega_{X}(t) ; \tilde{k}_{D_{X}}=k_{D_{X}}+\Delta k_{D_{X}} ; \Delta k_{D_{X}}>0 \\
& \widetilde{U}_{D_{X}}(t)=\widetilde{U}_{D_{X}}^{\max }(t)=\mathrm{const} \\
& \widetilde{U}_{D_{X}}(t)=\widetilde{U}_{D_{X}}^{\max }(t)=\mathrm{const}
\end{aligned}
$$

On the other hand, the arrangement on each axis of the three sensors aims to increase redundancy and ensure the system's safety in case of a problem. For sensors $\mathrm{S}_{0}$ lying on diagonal OO' has the form of the mathematical model as follows:

$$
\widetilde{U}_{0}(t)=\frac{1}{\sqrt{3}}\left[k_{D_{X}} \omega_{X}(t)+k_{D_{Y}} \omega_{Y}(t)+k_{D_{Z}} \omega_{Z}(t)\right]
$$

Where: $k_{D_{X}} \omega_{X}(t) ; \quad k_{D_{Y}} \omega_{Y}(t) ; \quad k_{D_{Z}} \omega_{Z}(t)$ - The output voltage of the sensors at the axes of the coordinate axis system.

From the mathematical and analytical models in the formulas from Eq. (1) to Eq. (8), we can observe that:

- There may be a malfunction on a sensor;

- There can be only one failure per sensors;

- Failures on each sensor are independent of each other;

- The output signal characteristics of the sensors are related to the failure modes that we have considered above;

- The output signal characteristic of the SO sensor is related to angular speed sensors on the axes of the link coordinate system.

Therefore, we can rely on the sensors' output signal characteristics to serve as a basis for detecting the failure of these sensors.

\section{Method}

The failure detection of a diagnostic object is done according to the following principle diagram:

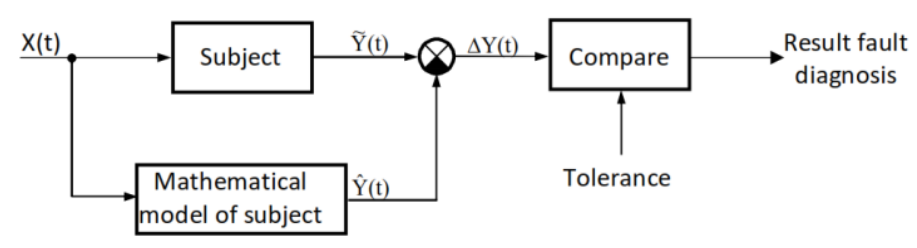

Fig.5. Principle of fault diagnosis

With the input value $\mathrm{X}(\mathrm{t})$ when passing the diagnostic object and its mathematical model, two different values are $\tilde{Y}(t)$ and $\hat{Y}(t)$. Let these two values through the subtractor, and we get the result $\Delta Y(t)$. Theoretically, the value $\Delta Y(t)=0$, but, this value is always different from 0 and is always less than something we call this value tolerance. Comparisons $\Delta Y(t)$ and tolerance will give us the failure detection results.

Applying the above principle, we assume that the $\mathrm{k}$ coefficient of the sensors is the same, so we have the mathematical model of the sensor on the following axes:

For the axis OX (Group X)

$\widetilde{U}_{x 1}(t)=k \omega_{X}(t) ; \widetilde{U}_{x 2}(t)=k \omega_{X}(t) ; \widetilde{U}_{x 3}(t)=k \omega_{X}(t)$

For the axis OY (Group Y)

$\widetilde{U}_{y 1}(t)=k \omega_{Y}(t) ; \widetilde{U}_{y 2}(t)=k \omega_{Y}(t) ; \widetilde{U}_{y 3}(t)=k \omega_{Y}(t)$

For the axis OZ (Group Z)

$\widetilde{U}_{z 1}(t)=k \omega_{Z}(t) ; \widetilde{U}_{z 2}(t)=k \omega_{Z}(t) ;$

$\widetilde{U}_{z 3}(t)=k \omega_{Z}(t)$

For the diagonal of the cube (OO') 
$\widetilde{U}_{0}(t)=k \frac{1}{\sqrt{3}}\left[\omega_{X}(t)+\omega_{Y}(t)+\omega_{Z}(t)\right]$

Relationships:

$\widetilde{U}_{m}=\frac{1}{\sqrt{3}}\left(\widetilde{U}_{X i}+\widetilde{U}_{Y j}+\widetilde{U}_{Z k}\right)$

Where:

$\mathrm{m}=1: 27 ; \mathrm{i}, \mathrm{j}, \mathrm{k}=1: 3$;

$\widetilde{U}_{m}$ - the output voltage value of block sensors;

$\mathrm{k}_{0}$ - the amplification coefficient of the sensors of the same type;

$\omega$ - the value of angular rate.

Thus, from the general relation expression (13), we have 27 specific relationships according to the indexes $\mathrm{i}, \mathrm{j}, \mathrm{k}$;

To build the algorithm to diagnose the state and damage angular speed sensing block based on the expression (13). The expression (13) show that the expression is correct in the ideal case, but in reality, despite the normal operating conditions of all expressions, the expression (13) has the form:

$$
\left|\widetilde{U}_{0}-\frac{1}{\sqrt{3}}\left(\widetilde{U}_{X i}+\widetilde{U}_{Y j}+\widetilde{U}_{Z k}\right)\right|
$$

Where: $\delta$ - the largest deviation under normal operating conditions, $\delta>0$.

When there is a failure error, the inequality (14) will not be true; that is $\left|\widetilde{U}_{0}-\frac{1}{\sqrt{3}}\left(\widetilde{U}_{X i}+\widetilde{U}_{Y j}+\widetilde{U}_{Z k}\right)\right|>\delta$. Thus, the conditions to determine a failure in the block can be set as follows:

Consider $\Delta_{n}=\left|\widetilde{U}_{0}-\frac{1}{\sqrt{3}}\left(\widetilde{U}_{X i}+\widetilde{U}_{Y j}+\widetilde{U}_{Z k}\right)\right|$, with $\mathrm{n}=1: 27$ is the ordinal number according to the combination options $\mathrm{i}, \mathrm{j}, \mathrm{k}$.

If $\Delta_{n}>\delta,(\mathrm{n}=1: 27)$ there is a failure in the sensors block.

If $\Delta_{n} \leq \delta$, (with all $\mathrm{n}=1: 27$ ), there is no failure in the sensor block.

Based on the above analysis, the improved angular speed sensor failure diagnostic algorithm is set up as follows:

The above algorithm only allows detecting and diagnosing a faulty sensor. However, it has not determined which sensor's failure because this sensor block has ten single sensors. Therefore, to determine precisely which fault sensor in the sensor block, we have developed an algorithm to determine each sensor's failure.

In the general case, there are three different fault groups on three axes. Unfortunately, the algorithm was built in the previous section does not meet the fault detection requirements on each sensor. Therefore, we need to build a general algorithm that can fault detection on each axis's sensors.

The typical virtual output values of diagonal sensor $\mathrm{S}_{0}$ are as follows:

$$
\widetilde{U}_{0 m}(k)=\frac{1}{\sqrt{3}}\left(\widetilde{U}_{X i}(k)+\widetilde{U}_{Y j}(k)+\widetilde{U}_{Z k}(k)\right)
$$

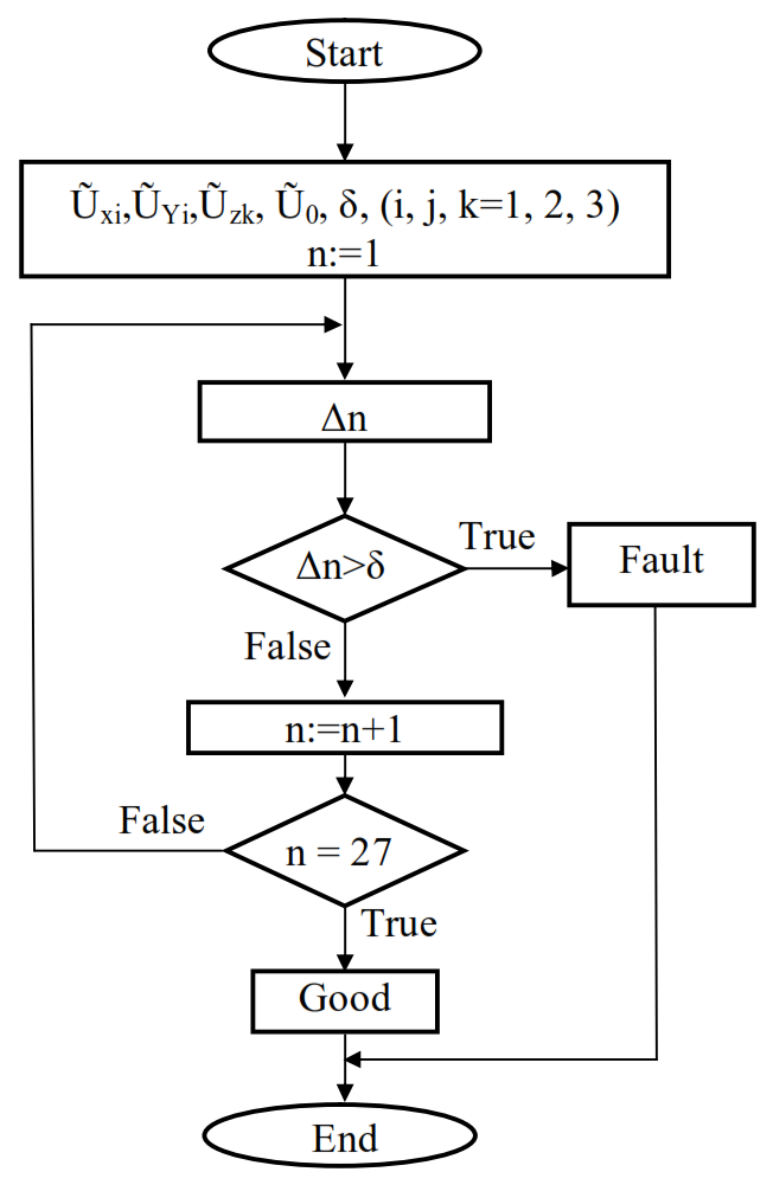

Fig. 6. Algorithm flowchart representing fault diagnostic algorithm improved angular rate sensors block.

Where: $\mathrm{m}$ - get values from 1 to 27 ;

$\mathrm{i}, \mathrm{j}$, k- get values from 1 to 3 ;

We will build a fault detection algorithm based on comparing the actual output value and the common virtual output value of the sensor $\mathrm{S}_{0}$.

$$
\begin{aligned}
Z_{m}=S_{m}\left\{\Delta_{0 m}=\right. & \left.\left|\widetilde{U}_{0}(k)-\widehat{U}_{0 m}(k)\right|>\delta_{0}\right\} \\
& =\left\{\begin{array}{l}
0-\text { not fault in sensors } \\
1-\text { fault in sensor } \mathrm{S}(\mathrm{i}, \mathrm{j}, \mathrm{k}) \text { or } \mathrm{S}_{0}
\end{array}\right.
\end{aligned}
$$

Based on equation (16), if there is 1 fault per sensor, then there are 9 values of $Z_{m}=1$, and if there are 2 failures on 2 sensors, there are 18 values of $Z_{m}=1$.

To facilitate the failure diagnosis in the general case, we set up a table of 27 values of $Z_{m}$ corresponding to the values of $i, j, k$, taking the following values from 1 to 3 , respectively:

From expression (16), we build a flow chart of fault diagnosis algorithm in the general case in fig 7.

Thus, with the general algorithm flowchart presented in Figure 7, we can diagnose the failure of angular speed sensors in 1 or 2 or all three groups. 
Table 1. Table of symbols of fault cases

\begin{tabular}{cccc}
\hline $\mathrm{Z}_{\mathrm{m}}$ & $\mathrm{U}_{\mathrm{X}}$ & $\mathrm{U}_{\mathrm{Y}}$ & $\mathrm{U}_{\mathrm{Z}}$ \\
\hline 1 & 1 & 1 & 1 \\
2 & 1 & 1 & 2 \\
3 & 1 & 1 & 3 \\
4 & 1 & 2 & 1 \\
5 & 1 & 2 & 2 \\
6 & 1 & 2 & 3 \\
7 & 1 & 3 & 1 \\
8 & 1 & 3 & 2 \\
9 & 1 & 3 & 3 \\
10 & 2 & 1 & 1 \\
11 & 2 & 1 & 2 \\
12 & 2 & 1 & 3 \\
13 & 2 & 2 & 1 \\
14 & 2 & 2 & 2 \\
15 & 2 & 2 & 3 \\
16 & 2 & 3 & 1 \\
17 & 2 & 3 & 2 \\
18 & 2 & 3 & 3 \\
19 & 3 & 1 & 1 \\
20 & 3 & 1 & 2 \\
21 & 3 & 1 & 3 \\
22 & 3 & 2 & 1 \\
23 & 3 & 2 & 2 \\
24 & 3 & 2 & 3 \\
25 & 3 & 3 & 1 \\
26 & 3 & 3 & 2 \\
27 & 3 & 3 & 3 \\
\hline & & &
\end{tabular}

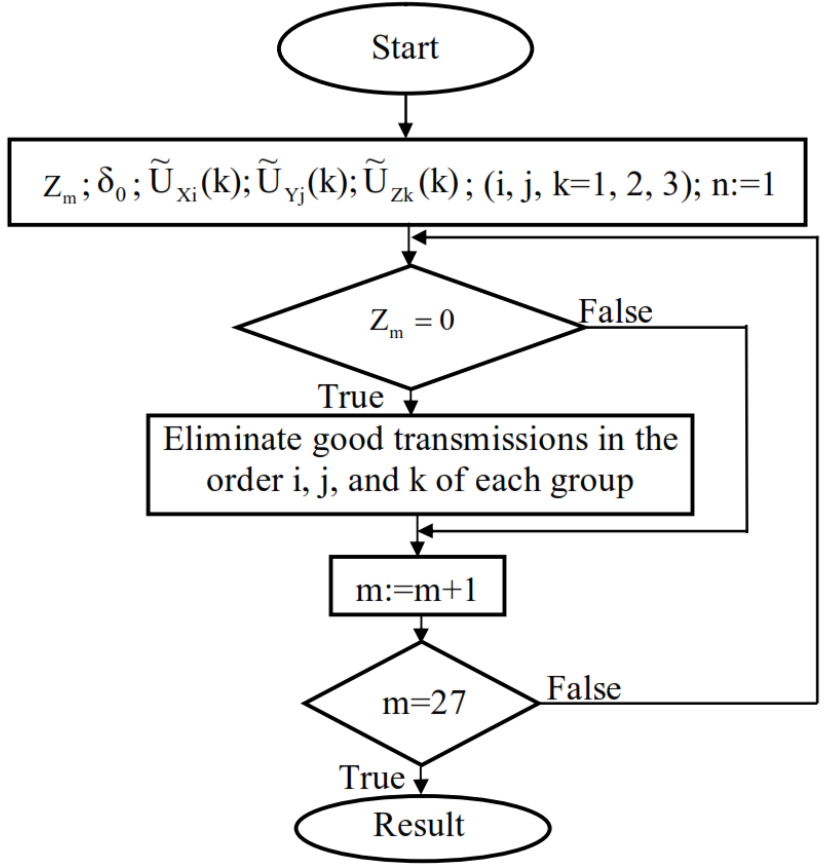

Fig. 7. Algorithm flowchart representing fault diagnostic algorithm improved angular rate sensor block in case of three group fault.

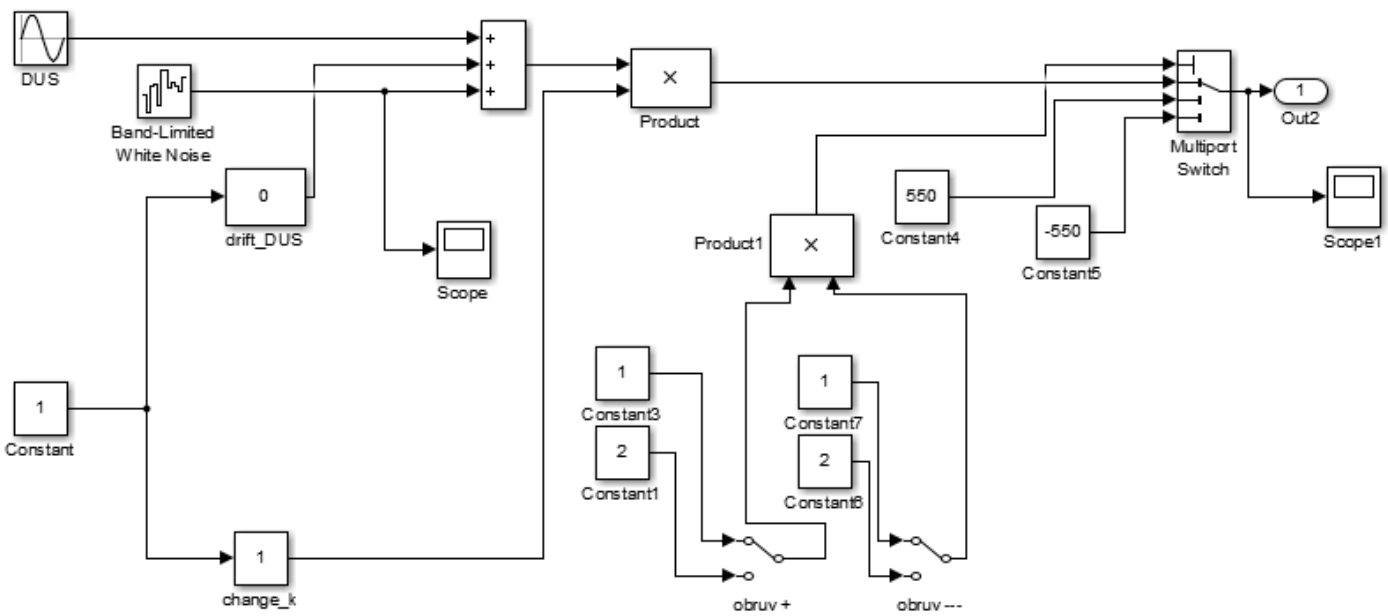

Fig. 8. Diagram simulation sensors block.

\section{Results}

The author uses the Matlab Simulink tool in Matlab software to simulate fault diagnosis algorithm in the general case.

The Simulink diagram simulates the operating principle of an angular speed sensor shown in Fig. 8.

The signal form of the sensor in the case of suitable working inductance is shown in the following Figs. 9-13.

With the characteristics of the output signal of the sensor simulated on Matlab Simulink, it is like the primary signal form of the actual angular speed sensor and like the mathematical model proposed by the author above.
A schematic diagram of the fault diagnosis algorithm in the case of 3 failure groups is shown in Fig. 14. The sensors block is calculated based on Eq. (2-8); the computational block is based on Eq. (16). Problem diagnosis block using State-Flow tool with 54 comparisons of fault diagnosis results shown at the output as a signal: 1- Fault sensor; 0-Good sensor.

A check on the algorithm's good operation shown in Fig. 5 is done by changing the sensors' parameters and seeing the sensors fail, and running the Matlab program to check the output parameters. Assumes sensors $\mathrm{X}_{2} ; \mathrm{Y}_{3} ; \mathrm{Z}_{2}$ fault and runs the simulation. We have the results shown in Fig. 15. 


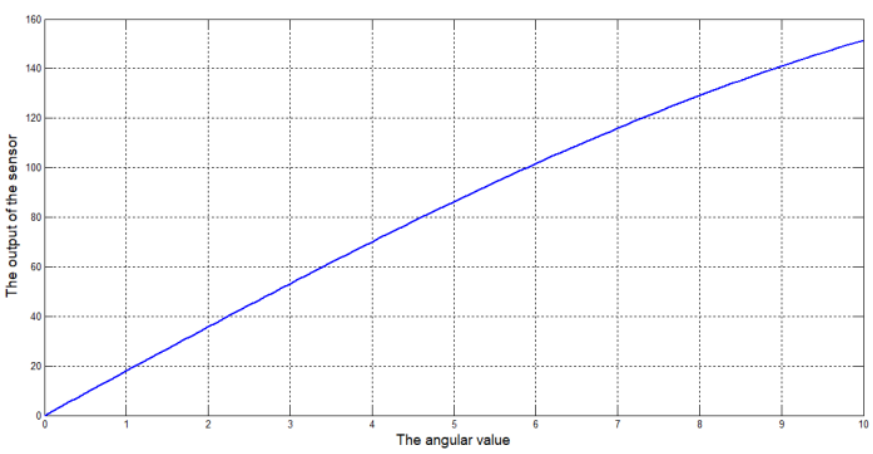

Fig. 9. Diagram showing the signal form of the good sensor.

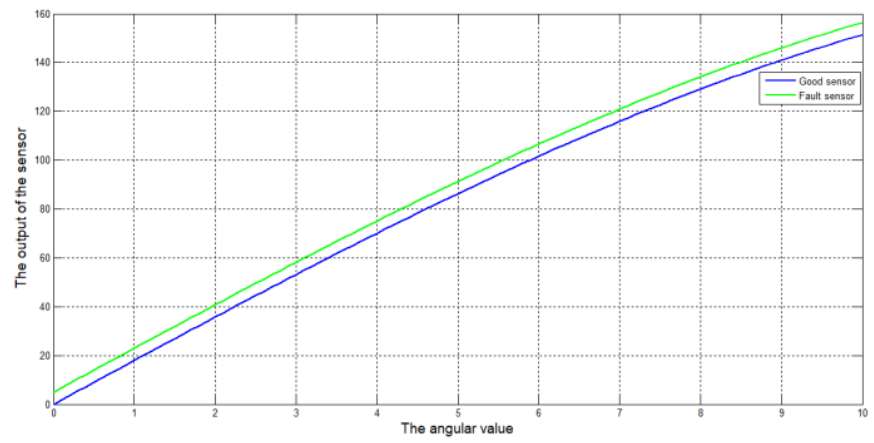

Fig. 10. The diagram comparing the signal form when the sensor has a signal drift problem.

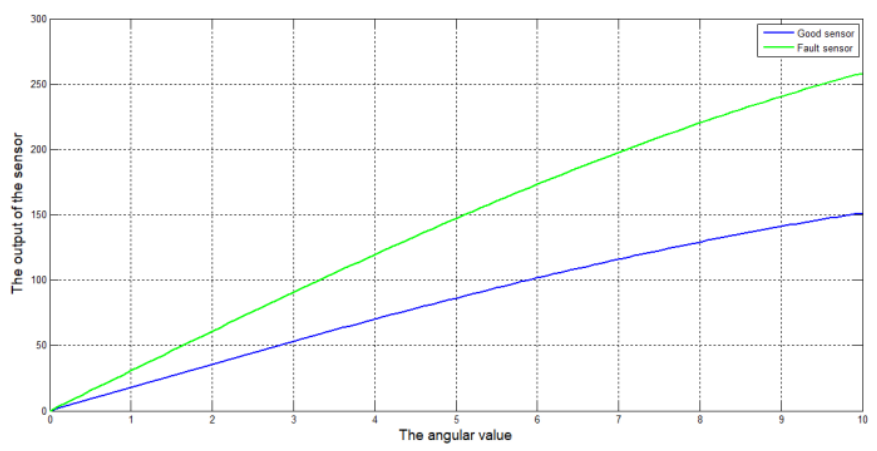

Fig. 11. The diagram compares the signal form when the sensor has a problem with the gain change.

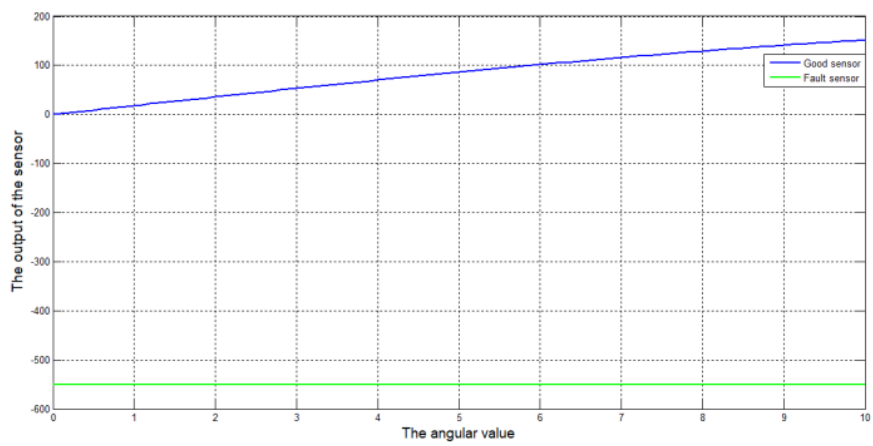

Fig. 12. The diagram compares the signal form when the sensor has a problem with the signal negative wire.

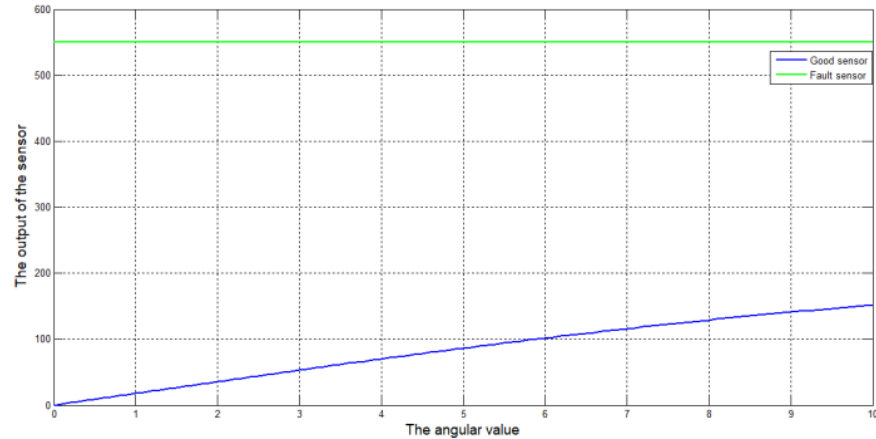

Fig. 13. The diagram compares the signal form the sensor has a problem with signal positive wire.

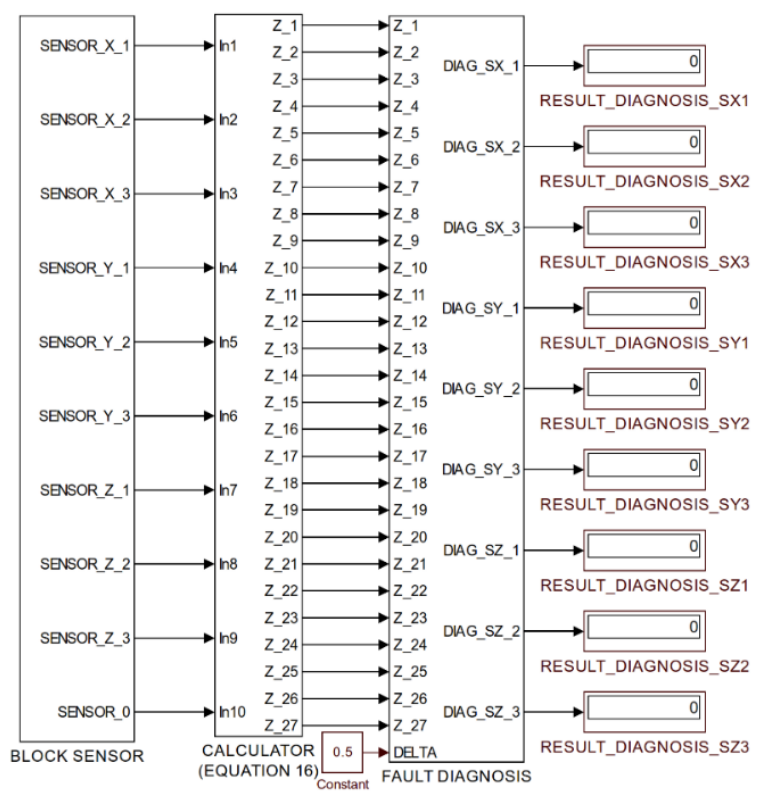

Fig. 14. Diagram simulation of fault diagnosis algorithm of improved sensors block.

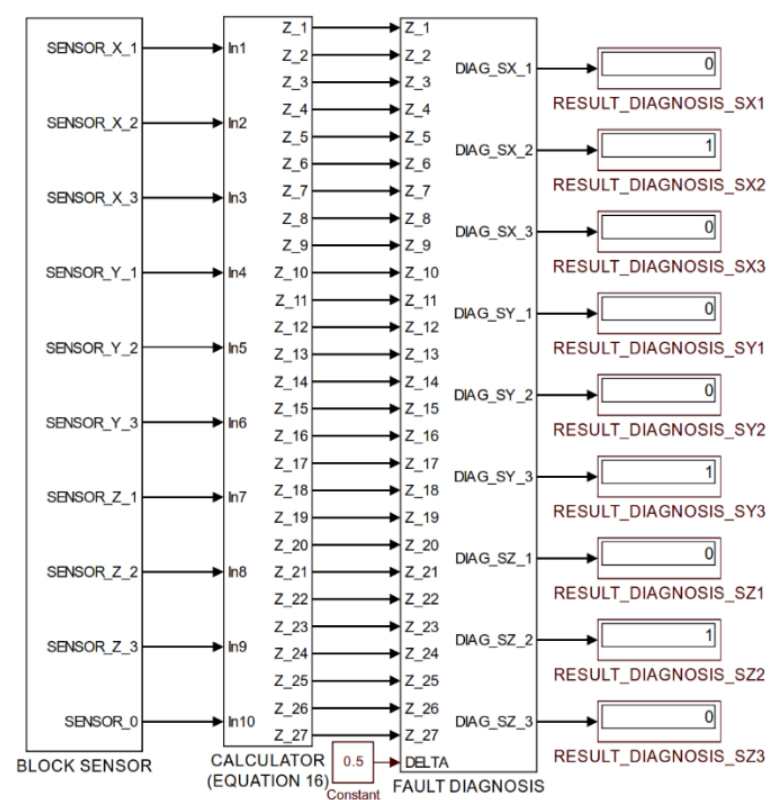

Fig. 15. Simulation results in case of fault sensors $X_{1}, Y_{3}$, $\mathrm{Z}_{2}$

After that, we assume that the sensors $\mathrm{X}_{2}, \mathrm{Y}_{2}, \mathrm{Y}_{3}$, and $\mathrm{Z}_{2}$ fail, and we will have the results in Fig. 8 . 


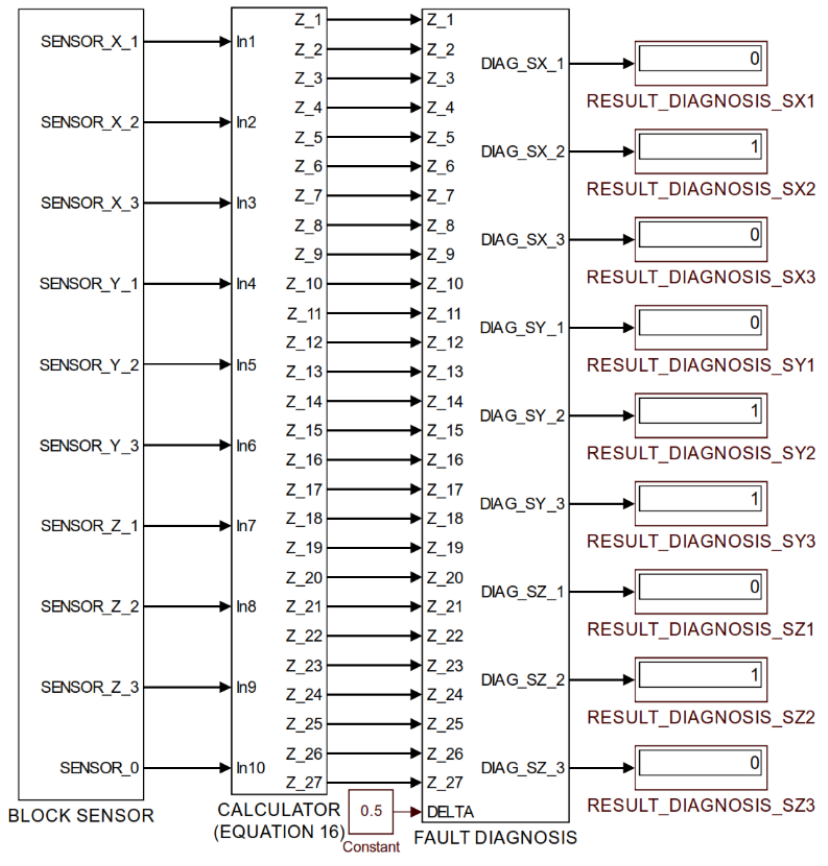

Fig. 16. Results in the case of fault sensors $X_{2}, Y_{2}, Y_{3}, Z_{2}$

\section{Conclusions}

In this paper, we developed a method for detecting and estimating the faults of angular velocity sensors in aircraft control systems, which can be used to expand block sensors' information. The simulation results show that the algorithm presented in Fig. 7 is entirely correct. Therefore, based on the results, we can expand the information of the angular velocity sensor. At this time, the output signal is an angular speed parameter and a failure signal of each sensor to warning the user.

Based on the sensors unit's information expansion method, it improves the control system's efficiency and ensures the safe operation of the aircraft based on multiple information sources. This method can be used for the same sensor in other systems in aircraft. However, this method stops the failure level. The subsequent studies will upgrade the algorithm to identify failure pattern and propose solutions to fix them.

\section{Abbreviations}

UAV : Unmanned Aerial Vehicle

FDD : : Fault Detection and Diagnosis

\section{CRediT Author Statement}

Hong Son Tran: Conceptualization, Methodology, Software, Investigation, Validation, Writing-Original Draft. Dinh Dung Nguyen: Conceptualization, Methodology, Writing-Review \& Editing, Visualization, Supervision. Thi Thuy Tran: Conceptualization, Methodology, Software, Investigation, Validation, Writing-Original Draft. Quoc Dat Dang: Conceptualization, Methodology, Software, Investigation, Resources, Writing-Original Draft. Hong
Tien Nguyen: Conceptualization, Methodology, Software, Resources, Investigation, Writing-Original Draft.

\section{References}

Xue, W., Guo, Y.Q. and Zhang, X.D., 2007, September. A bank of Kalman filters and a robust Kalman filter applied in fault diagnosis of aircraft engine sensor/actuator. In Second International Conference on Innovative Computing, Informatio and Control (ICICIC 2007) (pp. 10-10). IEEE.

He, Q., Zhang, W., Lu, P. and Liu, J., 2020, Performance comparison of representative model-based fault reconstruction algorithms for aircraft sensor fault detection and diagnosis. Aerospace Science and Technology, 98, p.105649.

Chen, J. and Patton, R.J., 2012, Robust model-based fault diagnosis for dynamic systems (Vol. 3). Springer Science \& Business Media.

Isermann, R., 2005. Model-based fault-detection and diagnosis-status and applications. Annual Reviews in control, 29(1), pp.71-85.

Lu, P., Van Eykeren, L., Van Kampen, E., De Visser, C.C. and Chu, Q.P., 2016, Adaptive three-step kalman filter for air data sensor fault detection and diagnosis. Journal of Guidance, Control, and Dynamics, 39(3), pp.590-604.

Lu, P., Van Eykeren, L., van Kampen, E.J., de Visser, C. and Chu, Q., 2015, Double-model adaptive fault detection and diagnosis applied to real flight data. Control Engineering Practice, 36, pp.39-57.

Kim, S., Choi, J. and Kim, Y., 2008, Fault detection and diagnosis of aircraft actuators using fuzzy-tuning IMM filter. IEEE Transactions on Aerospace and Electronic Systems, 44(3), pp.940-952.

Xue, W., Guo, Y.Q. and Zhang, X.D., 2007, September. A bank of Kalman filters and a robust Kalman filter applied in fault diagnosis of aircraft engine sensor/actuator. In Second International Conference on Innovative Computing, Informatio and Control (ICICIC 2007) (pp. 10-10). IEEE.

Baskaya, E., Bronz, M. and Delahaye, D., 2017, September. Fault detection \& diagnosis for small UAVs via machine learning. In 2017 IEEE/AIAA 36th Digital Avionics Systems Conference (DASC) (pp. 1-6). IEEE.

Hajiyev, C. and Soken, H.E., 2013, Robust adaptive Kalman filter for estimation of UAV dynamics in the presence of sensor/actuator faults. Aerospace Science and Technology, 28(1), pp.376-383.

Tuan, D. Q., Firsov, S.N. and Pishchukhina, O.A. "Design a fault diagnose block of angular velocity sensors for control systems of a multipurpose aircraft". Science and Technology of the Air Force of Ukraine, 2012, Vol. 11, issue 2, pp. 84-88. 